\title{
La exposición de arte desde la transferencia del conocimiento artístico
}

\author{
The exhibition of art from the transfer of artistic knowledge
}

Ana TIRADO. Universidad de Jaén (España).atirado@ujaen.es

Resumen: Este trabajo aborda la exposición de arte en clave de transferencia del conocimiento artístico. La hipótesis se refiere a si es posible un carácter científico para la exposición de arte y, en tal caso, cómo se articularía. Para ello, se adopta un método de estudio comparado de casos, de acuerdo a una muestra de dos casos internacionales de exposición de arte. Se realiza un análisis cualitativo de los contenidos de ambas exposiciones, según las variables de los dispositivos de información interna en sala y los contenidos de estos. Los casos de la muestra son: la exposición Itinerarios del vértigo de Sandra Silva, en el Museo de Arte de Pereira, en Pereira (Colombia), en 2015, y la exposición La invención concreta. Colección Patricia Phelps de Cisneros en el Museo Nacional Centro de Arte "Reina Sofía", en Madrid (España), en 2013. En los resultados se encuentran tres tipos de contenidos con carácter científico (crítica artística, metodología de investigación y fuentes primarias), respecto de un total de seis dispositivos de información interna en sala. Las conclusiones arrojan aportaciones para dos tipos de transferencia de conocimiento artístico en la exposición de arte, según la transferencia del proyecto artístico y la transferencia del contexto histórico-artístico.

Palabras clave: artes, museología, exposición, transferencia del conocimiento, investigación artística.

Abstract: This work addresses the exhibition of art in the key of transfer of artistic
knowledge. The hypothesis refers to whether a scientific character is possible for
the art exhibition, where the main purpose is to know how this transfer would be
articulated in an art exhibition. For this, a method of comparative study of cases
is adopted, with a sample of two international cases of art exhibition, in which a
qualitative analysis of contents of the exhibition is carried out, according to the 
variable of the internal information devices in the room and the contents of these. The cases of the sample are: the exhibition Itinerarios del vertigo by Sandra Silva, at the Art Museum of Pereira, in Pereira (Colombia), in 2015, and the exhibition of Concrete Invention. Collection of Patricia Phelps de Cisneros, at the National Museum Center of Art "Reina Sofía", in Madrid (Spain), in 2013. In the results, three different types of content are found (artistic criticism, research methodology and primary sources), according to a total of six internal information devices in room. The conclusions provide contributions for two types of transfer of artistic knowledge in art exhibition, according to the transfer of the artistic project and the transfer of the historical-artistic context.

Keywords: art, museology, exhibition, transfer of knowledge, art research.

\section{Introducción}

Este trabajo aborda la exposición de arte en clave de transferencia del conocimiento. La transferencia tiene como objetivo incorporar el conocimiento a una cadena de valor para que genere un retorno económico (Universidad Autónoma de Barcelona, 2018). Anteriormente, las acciones en materia de transferencia estuvieron concentradas en la aplicación tecnológica, pero, a partir de los años noventa del siglo XX y ante el nuevo contexto de las economías y sociedades basadas en el conocimiento, comenzó a normalizarse la transferencia del conocimiento con un sentido más amplio y englobando todas las áreas (Castro et al., 2008).

El conocimiento artístico concierne a cuestiones epistemologías de la producción artística en su conjunto. La creación artística sucede en medio de procesos del conocimiento que tiene el objetivo de crear sentidos sobre una realidad dada (Arañó, 2005), así como de la interacción y del diálogo socio-cultural de los valores y concepciones que, sobre el entorno, tienen determinados grupos sociales y las personas que participan de ello (Guash, 2016; Conderana, 2016). El comisariado de exposiciones de arte realiza un intenso trabajo de investigación, documentación y análisis crítico de las obras, los/as artistas y sus contextos históricos y actuales. Cabe entonces preguntarse dónde queda este compendio de información y conocimiento artístico. Comúnmente, parte de esta información y conocimiento constituyen los contenidos de audio-guías, aplicaciones digitales, material digital en línea y, más tradicionalmente, constituyen el catálogo de la exposición. Así, tradicionalmente, el material científico generado en torno a las obras y la exposición queda a expensas del montaje expositivo, esto es, fuera de las salas de exposición, utilizando medios $\mathrm{y}$ recursos de difusión de esta información que no sea intrusivos con el campo visual de las obras.

Respecto de una transferencia del conocimiento artístico en la exposición de arte, se entiende que las formas de exponer el arte son deudoras de los modos de pensarlo. En palabras de Dubé: “(...) las maneras de exponer son tributarias de las maneras 
de pensar la exposición, [dado que] la ciencia, por su riguroso método de examen empírico, va a influir enormemente en los modos de exposición museística gracias a su enfoque constantemente renovador de la materia estudiada" (Dubé, 1995, p. 5). Dentro de las salas, algunas innovaciones se han dirigido a la inclusión en sala de señalética de rótulos con preguntas (Lorente, 2013) y a explotar las cualidades escenográficas y sensitivas de la exposición, como Pérez (2007), quien trabaja sobre la "exposición creativa", interviniendo sobre muros, techos, etc., con elementos pictórico-gráficos para intensificar la experiencia estética de las obras en sala.

\section{Hipótesis y objetivos}

La hipótesis de este trabajo se refiere a si se puede reconocer una tipología científica para la exposición de arte. Alonso (2001, p. 202) distingue cuatro tipologías de exposición, según las siguientes: la simbólica, la comercial, la documental y la estética. Siguiendo al autor, la exposición estética se refie a a los museos y galerías respecto de un formato de exposición donde las obras constituyen el único elemento. Sin embargo, el tipo de exposición documental, que se refire al valor informativo o científico de los objetos, está asociado a museos de carácter científico o técnico, como los ecomuseos, por ejemplo -según cita el autor-. De acuerdo a una tipología estética de la exposición de arte, esta responda al formato de galería de arte, según una museografía de muros lisos y neutros (Lorente, 2008; Rico, 2007; Ávila, 2003; Alonso, 2001; Hooper-Greenhill, 1992). Este formato es heredero del legado históriográfico de la historia del arte de Winckelmann, cuando a mediados del siglo XVIII protagonizó el giro historiografíco de la historia del arte, anteriormente focalizada sobre los artistas y sus planteamientos de producción del arte y, desde entonces, dirigida hacia las cualidades estéticas de las obras por sí mismas (Winckelmann, 1763). Sin embargo, los discursos artísticos contemporáneos y actuales han superado el fenómeno estético de la obra por un fenómeno cultural de la produccion artística. Entonces ¿por qué las exposiciones de arte se mantienen como mausoleos de objetos? ¿No se podría plantear la exposición de arte también en clave de difusión científica del conocimiento? ¿Cómo podría hacerse? Otras museologías han alcanzado este tratamiento científico de sus exposiciones, como los museos de ciencias, donde es común que los montajes de exposiciones en sala atiendan las cuestiones de qué es esto, de qué se compone, para qué sirve y cómo se utiliza y, por tanto, que los/as visitantes interactuen desde el primer momento con muy diversos dispositivos dentro de la exposición misma: cédulas, gráficos, vitrinas, vídeos, aparadores, “displays”, etc. (Rojas, 2007). Por tanto, el objetivo de este trabajo es conocer cómo se articula esta transferencia del conocimiento artístico en la exposición de arte, para un formato de exposición con sentido científico.

\section{Método}

Para conocer cómo se articula esta transferencia del conocimiento artístico en la exposición de arte, se adopta un método de estudio comparado de casos (Masseroni, 
2010), según una muestra de dos casos internacionales de exposición de arte que son representativos de integrar dispositivos en sala alusivos a la producción artística de la obra. De este modo, el análisis del estudio está dirigido a conocer cuáles son los contenidos que estos dispositivos introducen en sala y si estos suponen una aportación al carácter científico de la exposición de arte. Se definen dos variables para el análisis, según los dispositivos museográficos internos en sala y según los contenidos de estos mismos dispositivos. Debido a la variedad tipológica de los dispositivos en sala entre los casos y a que constituyen una elaboración original de cada montaje expositivo, el análisis del estudio se aborda de forma deductiva. Para delimitar los dispostivos que se analizan se establece que son dispositivos de estudio los elementos museográficos internos en sala de las exposiciones, que contengan información relativa a las obras, sus autores, historia y producción artística, sin discriminar por tipología del formato (señalética, vídeo, gráfico...) ni lenguaje empleado (escrito, visual, etc.).

\section{La muestra}

La muestra abarca dos casos internacionales de exposición de arte, que son:

- La invención concreta. Colección Patricia Phelps de Cisnteros (IC-CPPC en adelante), expuesta en el Museo Nacional de Arte Contemporáneo Reina Sofía, en Madrid (España), del 23 de enero al 16 de septiembre de 2013, con obras procedentes de la Colección venezolana Patricia Phelps de Cisneros.

- Itinerarios del vértigo (It $V$ en adelante), expuesta en el Museo de Arte de Pereira, en Pereira (Colombia), del 26 de junio al 16 de agosto de 2015, con obra de la artista Sandra Silva.

El criterio de selección ha sido el de exposiciones de arte que dispongan de dispositivos museográficos de información interna en sala. Entre las características de la muestra destaca el ámbito internacional, según dos museos con sede en Madrid (España) y Pereira (Colombia), respectivamente. Esto manifiesta que el tema de estudio del carácter científico de la exposición de arte no se trata de una cuestión aislada ni localizada en focos concretos, sino que más bien se refiere a una cuestión global del ámbito museológico del arte. Otra característica es la periodicidad reciente y actual de los casos de la muestra, según una cronología de los años 2015 y 2013, respectivamente. A continuación se describe la muestra, según cada caso de exposición de arte.

Exposición La invención concreta en el Museo Nacional Centro de Arte "Reina Sofía" de Madrid (España).

La $I C-C P P C^{1}$ se trató de una exposición colectiva dedicada a artistas latinoamericanos

1. Portal web oficial de la exposición La invención concreta. Disponible en: http://www.lainvencionconcreta.org/es 
del movimiento artístico internacional de la abstracción geométrica, en el MNCARS. Las obras de la muestra tuvieron una periodicidad de entre los años treinta y sesenta del siglo XX. Abarcó un total de 114 obras, que correspondían con una treintena de artistas diferentes, como Juan Alberto Molenberg, Hélio Oiticica, Lygia Clark, entre muchos/as otros/as (Rodríguez y Anglès, 2013). Estuvo ordenada según cinco bloques temáticos principales (diálogo, universalismo, geometría, ilusión y vibración). El compendio de obras procedían de la colección privada de la Fundación Patricia Phelps de Cisneros (Venezuela). De este modo, esta exposición es resultado de una colaboración curatorial conjunta entre esta Fundación y el MNCARS ${ }^{2}$. En la sede en Madrid estuvo ubicada en la planta baja del espacio temporal del Edificio Nouvel del MNCARS -anexo a la sede principal del museo-.

Las obras expuestas fueron de tipo pintura y escultura de gran formato, aunque el intenso juego óptico de las obras, junto con sus dimensiones, expandían sus efectos visuales sobre el espacio - como las obras de Jesús Soto-. La señalética de la exposición consistió en un texto de presentación y las cartelas de identificación de cada una de las obras. El texto de presentación estuvo colocado en una sala que antecedía a la exposición, a modo de entrada; contuvo dos rótulos titulados "EXPLORAR E INTERACTURAR" y "DESCUBRIR Y COMPARTIR", que aludían a una propuesta participativa del público en la exposición, con referencias concretas al portal web de la exposición. "Interactuar" aludía a la actividad de publicar libremente por parte del público imágenes de la exposición en redes sociales de internet. Las cartelas contuvieron datos de título, año, autor y técnica de cada una de las obras, respectivamente; estuvieron elaboradas en texto negro sobre fondo blanco, mimetizadas con el muro, y colocadas junto a cada obra, respectivamente.

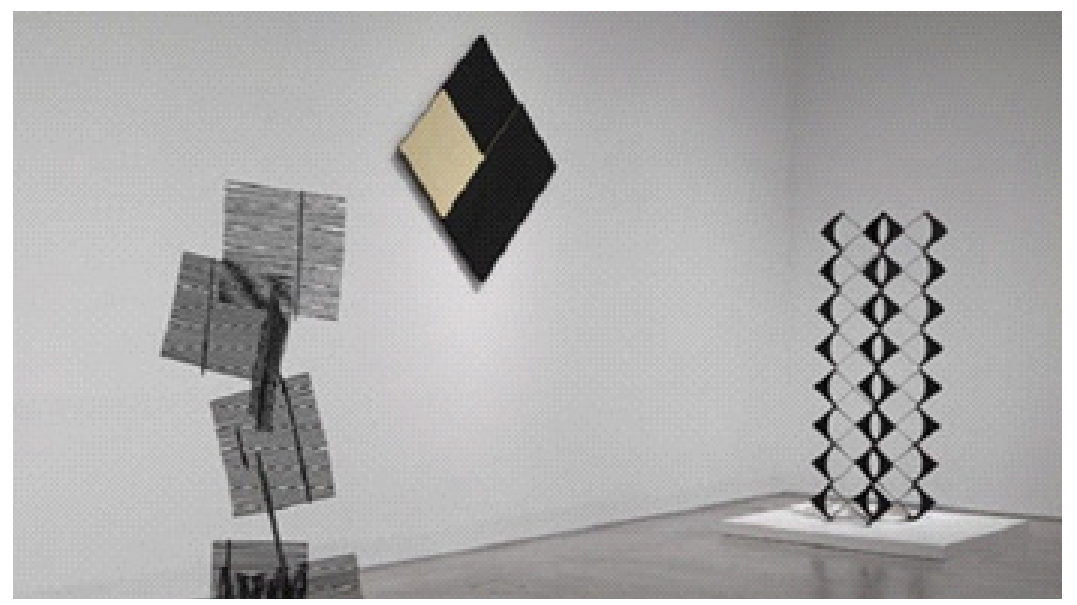

Figura 1. Vista de sala de la exposición. La invención concreta. Colección Patricia Phelps de Cisneros en el MNCARS en 2013. Fuente: MNCARS, 2013. Disponible en: https://www.museoreinasofia.es/ exposiciones/invencion-concreta-coleccion-patricia-phelps-cisneros

2. Página web dedicada a la exposición La invencinó concreta en el portal web oficial del MNCARS. Disponible en: http:// www.museoreinasofia.es/exposiciones/invencion-concreta-coleccion-patricia-phelps-cisneros 
En mitad del recorrido por las salas de la exposición, se encontraba una sala aparte dedicada a un diagrama mural. Esta sala era rectangular y diáfana. En su interior había dispuestas tres mesas blancas alargadas con aparatos electrónicos de iPads donde poder consultar y navegar por la aplicación digital de la exposición (gratuita), para sistemas operativos de iPad y de iPhone, respectivamente. También se encontraban libros especializados en la temática de la exposición para su consulta. El diagrama mural se refiere a la representación gráfica que se extendía a lo largo de los cuatro muros de la sala. Estuvo compuesto por formas circulares, palabras clave y líneas. Las formas circulares ordenaban visualmente los contenidos, cuya estructura seguía los cinco bloques temáticos principales de la exposición. Las líneas se utilizaban para unir visualmente entre palabras y fragmentos clave y, así, representar la afinidad temática y las relaciones de influencia entre sí. Las palabras clave eran de nombres de artistas de la exposición, así como de otras personalidades científicas e históricas y de movimientos artísticos que habían sido de influencia sobre estos artistas y su producción artística. En conjunto, este diagrama mural representaba gráficamente una síntesis de las referencias teóricas, históricas, científicas y artísticas principales para la producción artística de las obras expuestas.

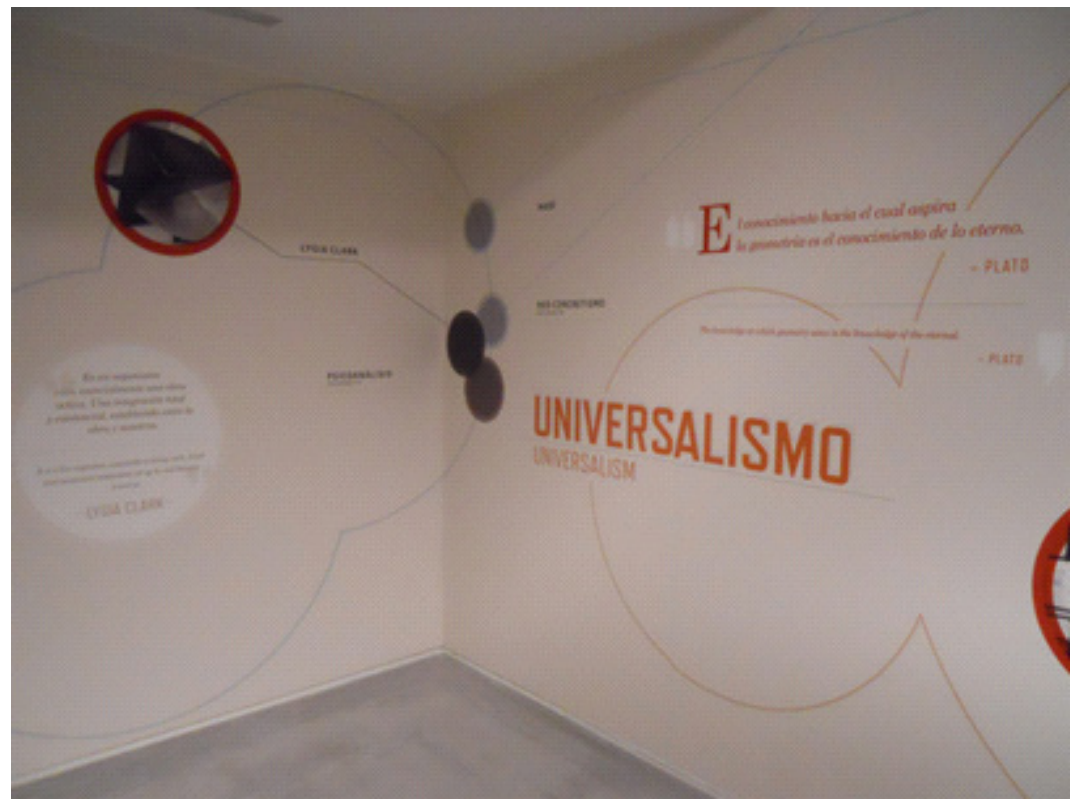

Figura 2. Vista del diagrama mural en la exposición La invención concreta. Colección Patricia Phelps de Cisneros en el MNCARS en 2013. Fuente: imagen de la autora, 2013.

Un ejemplo del diagrama mural es el bloque temático de DIÁLOGO, con el ejemplo de la artista Lygia Clark, y que estuvo relacionado con el filósofo Henri Focillon, la corriente de pensamiento del psicoanálisis y el estilo artístico del neo-concretismo. Incluye dos citas de ambos: de Lygia Clark, "Es un organismo vivo, esecialmente una obra activa. Una integración total y existencial, establecida entre la obra y nosotros"; y de Henri Focillon, "Estamos totalmente justificados en nuestra suposición de que tales formas constituyen y ordenan la existencia y que este orden tiene el movimiento y el suspiro de vida. Las formas plásticas están 
sujetas a el principio de la metamorfosis...". Siguiendo este mismo planteamiento de referencias, se elabora también para los otros cuatro bloques temáticos en los que se ordena el diseño curatorial de la exposición.

La colección de arte de la Fundación Patricipa Phelps de Cisneros ${ }^{3}$ constituye una de las colecciones privadas más importantes, con sede en Caracas y Nueva York. Tiene especial dedicación a artistas latinoamericanos. Esta Fundación viene desarrollando una importante labor educativa y didáctica del arte. Ejemplo de esta labor son el diagrama mural y la aplicación móvil que también acompañaron el proyecto expositivo en el MNCARS. El MNCARS constituye el museo nacional de arte contemporáneo de España, con sede en Madrid. Tiene un papel destacado en la conservación y difusión del arte español e iberoamericano. Resultado de esta política cultural nacional son las itinerancias entre el MNCARS con otras instituciones del otro lado del Atlántico ${ }^{4}$, de lo que también es ejemplo esta exposición de $\mathrm{La}$ invención concreta con la Fundación Patricia Phelps de Cisneros.

\section{Exposición Itinerarios del vértigo en el Museo de Arte de Pereira (Colombia)}

La It $V$ se trató de una exposición individual de Sandra Silva, que pudo verse en el Museo de Arte de Pereira ${ }^{5}$ (Colombia). La muestra artística estuvo compuesta por una serie de artefactos y obras de tipo instalación, audiovisual y libro de artista, en torno al tema del miedo y el colectivo travesti en la ciudad de Pereira. La obra audiovisual se tituló Crónica audiovisual "Días de Afrodita", de género documental, que estuvo basada en una recopilación de fragmentos audiovisuales de los lugares y locales de Pereira que las personas travesti frecuentan diariamente. La obra de instalación estuvo compuesta por una serie de objetos de maniquíes ataviados de acuerdo a las identidades de corporeidad y representación visual del colectivo travesti, y que fue titulado Vestidos Orales. El Libro de pensamiento y producción estético-artístico constituye la memoria de la investigación artística, donde se presentan las descripciones en texto escrito con las composiciones visuales de dibujos; este estuvo elaborado a modo de libro de artista.

Por su parte, los artefactos consistieron en una serie de objetos que la artista había elaborado para diferentes fases y tratamientos de su proyecto artístico y, aunque no constituyeron en sí mismos obras de la muestra expuesta, estos también fueron incluidos en la exposición. Estos artefactos fueron: el Mapa simbólico del miedo

\footnotetext{
3. Portal web oficial de la Fundación Patricia Phelps de Cisneros. Disponible en: https://www.coleccioncisneros.org/es

4. Página web de Itinerancias en el portal web oficial del MNCARS. Disponible en: https://www.museoreinasofia.es/ itinerancias/

5. Museo de Arte de Pereira. Sandra Silva: Itinerarios del vértigo [archivo de vídeo]. Youtube, 18 de julio de 2015. Disponible en: https://www.youtube.com/watch?v=evrv32F-_Xk
} 
y el Fobófono. El primero se trata de un mapa callejero de la ciudad de Pereira impreso a color sobre vinilo y tabla; sobre este, se habían utilizado pegatinas icónicas alusivas a diferentes tipos de miedo de la ciudad (robo de carros, atracos, indigencia, cosquilleo, venta y tráfico de drogas, entre otros), y que marcaban sobre el mapa las zonas de estos miedos, respectivamente; el mapa estuvo acompañado por un cartel en el que podía leerse: "Señale aquí los luguares más miedosos o peligrosos de la ciudad de Pereira”. El Fobófono, por su parte, da nombre a un aparato electrónico de registro de voces y el que se habían grabado testimonios orales de opinión y de historia de vida de la población de Pereira acerca de sus experiencias de miedo en la ciudad. En la exposición podían escucharse estos testimonios a través de unos auriculares. El Fobófono estuvo acompañado por otro cartel, que decía: “¿Qué es lo que le produce miedo en el sector del Parque de la Libertad?" Por tanto, ambos artefactos fueron diseñados y empleados por parte de la artista para intervenciones artísticas participativas con la población de Pereira y recoger sus testimonios de experiencias en torno al tema del miedo en la ciudad.

Otro de los elementos museográficos insertados en sala en esta exposición se trató de un mapa conceptual sobre uno de los muros. En concreto, este se dispuso en uno de los muros de la entrada a la exposición. Estaba compuesto por palabras clave y ordenadas entre sí a modo de esquema visual. La parte central y concéntrica indicaba "INVESTIGACIÓN BASADA EN LA CREACIÓN"; arriba, "ITINERARIOS DEL VÉRTIGO; debajo, "A partir de LO RELACIONAL y LO CONTEXTUAL"; en los laterales, "ACONTECIMIENTOS ESTÉTICO-ARTÍSTICOS", junto a "1.INSTALACIÓN VESTIDOS ORALES", "2.CRÓNICA AUDIOVISUAL DÍAS DE AFRODITA" y "3.LIBRO DE PENSAMIENTO Y PRODUCCIÓN ESTÉTICO-ARTÍSTICO"; y, también en los laterales, "PROVOCACIONES ESTÉTICO ARTÍSTICAS, junto a "1.MAPA SIMBÓLICO DEL MIEDO", “2.FOBÓFONO” y "3.REGISTRO AUDIOVISUAL VESTIDOS ORALES”. De este modo, este mapa conceptual reunía el total de cuestiones principales, obras y artefactos de la exposición y servía a modo de síntesis gráfica de esta.
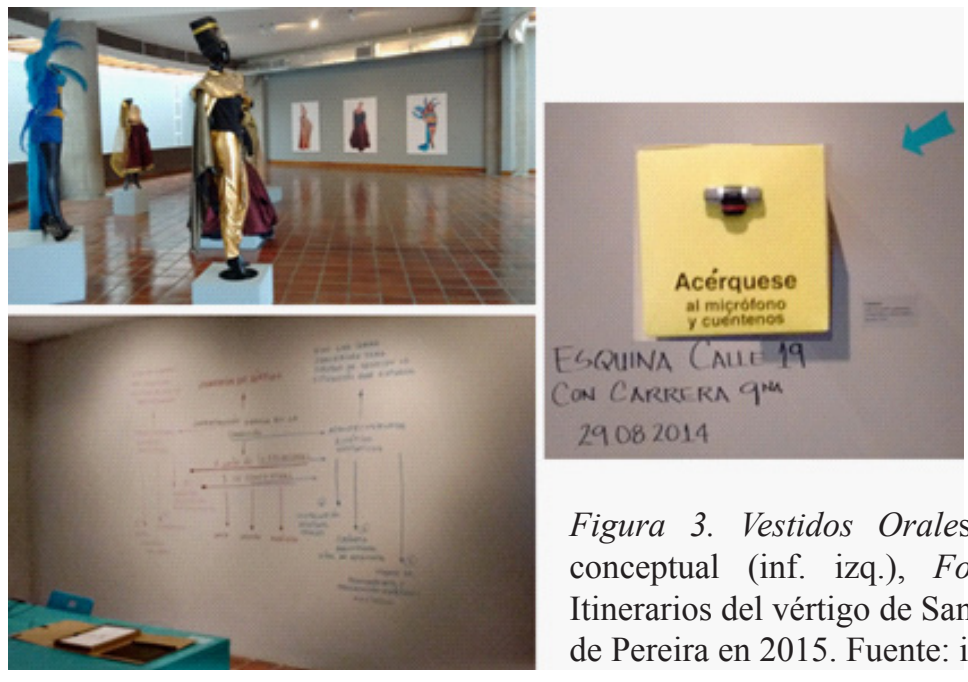

Figura 3. Vestidos Orales (imagen sup. Izq.), mapa conceptual (inf. izq.), Fobófono (dcha.). Exposición Itinerarios del vértigo de Sandra Silva en el Museo de Arte de Pereira en 2015. Fuente: imágenes de la autora, 2015. 
La señalética de la exposición consistió en un texto de presentación sobre el primero muro de la entrada, un texto de créditos y las cartelas identificativas de cada una de las obras y artefactos expuestos. El texto de presentación fue escrito por D. Adolfo León Grisales Vargas, Director Doctorado en Diseño y Creación de la Universidad de Caldas, en cuyo marco de estudios de postgrado la artista realizó la obra. El texto se refiere a una teoría crítica del Director sobre la investigación de Sandra Silva, aludiendo a tres aspectos: el miedo atraviesa toda la ciudad y no se refiere a rincones concretos; el miedo ha conducido a la población a recluirse en espacios privados de interior, de modo que el espacio exterior de la ciudad ha quedado deshabitada de señales de identidad; la desconfianza que existe entre la población respecto del colectivo travesti y que el Director relaciona con cierto recelo o envidia porque se muestran tal y como son. El texto de créditos indica la relación de autoría para los trabajos de producción y postproducción de las obras y sus diferentes elementos. Y las cartelas se restringen a los datos de título, año y técnica de elaboración de la obra.

El Museo de Arte de Pereira ${ }^{6}$ constituye el museo local y municipal de la ciudad de Pereira, con especial dedicación a la exposición de obras de carácter estético y visual. Está dirigido a artistas y/o temáticas regionales. En esta ocasión, este se presentaba como el lugar de mayor afinidad para exponer el trabajo artístico de ItV, según la artista había realizado una investigación local en la ciudad (el miedo en Pereira).

\section{Procedimiento}

Entre los dos casos de exposición de arte de la muestra se distinguen los siguientes dispositivos museográficos de información interna en sala:

- La señalética, según textos de presentación de la exposición y cartelería de identificación de las obras (correspondencia en los dos casos de la muestra).

- Mapa conceptual sobre el muro, Mapa simbólico del miedo y Fobófono (correspondencia solo en ItV.

- Diagrama mural (correspondencia solo en IC-CPPC).

Para la recogida de datos y debido al carácter físico y visual de la muestra, se realizó una observación directa (Angrosino, 2012; Díaz, 2012) en los sitios de las exposiciones, respectivamente, y empelando técnicas de grabación fotográfica y videográfica ${ }^{7}$. Para la recogida de datos en la exposición $I C-C P P C$ en el MNCARS, la investigadora realizó una residencia de una semana en septiembre de 2013, con permiso concedido por el Departamento de Educación del Museo. Para la exposición de ItV en el Museo de Arte de Pereira, la investigadora realizó varias

6. Portal web oficial del Museo de Arte de Pereira. Disponible en: https://museoartepereira.org/

7. La grabación fotográfica y videográfica estaba permitida libremente en ambos lugares de la muestra. 
y sucesivas visitas durante el periodo de estancia internacional de investigación de tres meses que realizó invitada por la Universidad del Valle, en Cali (Colombia) de mayo a julio de 2015. Para analizar el material fotográfico y videográfico recogido, se adopta un análisis de datos de tipo cualitativo, basado en principios de reducción de datos y utilizando procedimientos de clasificación de los datos (Medina et al., 2017; Miles, Huberman y Saldaña, 2014). Para clasificar los datos, se utiliza una herramienta de matriz, que se define del siguiente modo: en columnas verticales se distribuyen los tipos de contenidos y en filas horizontales los dispositivos internos en sala del análisis.

\section{Tabla 1}

Matriz de clasificación de datos del análisis.

CONTENIDOS

\begin{tabular}{|c|c|c|c|c|}
\hline EXPOSICIÓN & $\begin{array}{c}\text { DISPOSITIVOS } \\
\text { INTERNOS EN } \\
\text { SALA }\end{array}$ & Contenido 1 & Contenido 2 & Etc. \\
\hline $\begin{array}{c}\text { Itinerarios del } \\
\text { vértigo (ItV) }\end{array}$ & $\begin{array}{c}\text { Texto de } \\
\text { presentación } \\
\text { Cartelas } \\
\text { Mapa conceptual } \\
\text { sobre el muro } \\
\text { Mapa simbólico } \\
\text { del miedo } \\
\text { Fobófono }\end{array}$ & & & \\
\hline $\begin{array}{l}\text { La invención } \\
\text { concreta (IC- } \\
\text { CPPC) }\end{array}$ & $\begin{array}{c}\text { Texto de } \\
\text { presentación } \\
\text { Cartelas } \\
\text { Diagrama mural }\end{array}$ & & & \\
\hline
\end{tabular}

Fuente: elaboración de la autora, 2018.

Resultados

Los resultados del análisis están dirigidos a los tipos de contenidos respecto de los seis dispositivos de información interna en sala del análisis. Se distinguen un total de seis tipos diferentes de contenidos. Estos son:

1) Contenidos de crítica artística.

2) Contenidos de metodología de investigación.

3) Contenidos de contexto teórico y artístico.

4) Contenidos de fuentes primarias.

5) Contenidos de servicios al público.

6) Contenidos de identificación de las obras expuestas. 
La correspondencia de los cinco tipos de contenidos anteriores entre los seis dispositivos internos en sala analizados es la siguiente:

1. Los contenidos de crítica artística corresponden al dispositivo de señalética de texto de presentación de la exposición ItV.

2. Los contenidos de metodología de investigación corresponden al dispositivo de mapa conceptual de $I t V$.

3. Los contenidos de contexto teórico y artístico corresponden al dispositivo de diagrama mural de $I C-C P P C$.

4. Los contenidos de testimonios originales corresponden al dispositivo de Mapa visual del miedo y de Fobófono de It $V$.

5. Los contenidos de servicios al público corresponden con el dispositivo de señalética de texto de presentación de $I C-C P P C$.

6. Los contenidos de identificación de las obras corresponden con el dispositivo de señalética de cartelas, presente en ambos casos de exposición de la muestra.

Tabla 2

Resultados de tipos de contenidos y su correspondencia con los dispositivos en sala analizados de la muestra

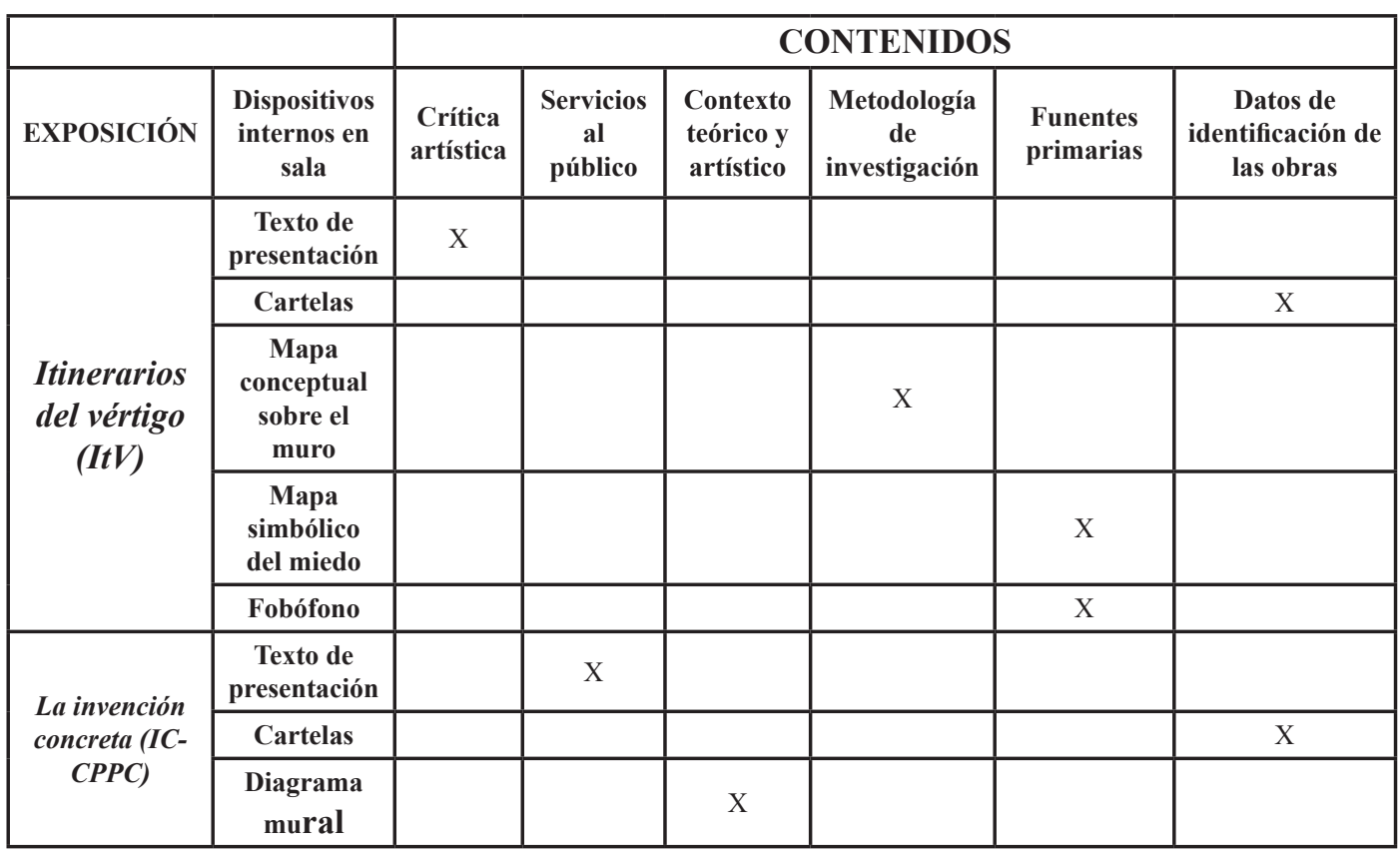

Fuente: elaboración de la autora, 2018.

Los contenidos de crítica artística consisten en juicios de valor, de opinión argumentados en torno al arte. El texto de presentación de It $V$ destaca cuáles son las aportaciones princiaples de la exposición al tema de las obras. Cuado se dice: "Uno de los aspectos más destacados del recorrido, de la investigación y apuesta de Sandra Silva con este trabajo (...) y vamos con recelo (o con envidia) a quienes 
se muestran como son: el travesti en realidad no se oculta detrás del maquillaje y la ropa que usa, sino que más bien es el único de nosotros que así logra mostrarse y exponerse como es", se tratan de ideas conceptuales y abstractas que el director de doctorado (quien lo escribe) quiere destacar, a modo de crítica artística.

La metodología de investigación consiste en la definición de un orden de pautas procedimentales que se establece de forma justificada y argumentada para el estudio y análisis de un tema dado. Comprende, desde la definición de una hipótesis o tema de estudio, la definición de objetivos, la identificación de fuentes principales de consulta y el método de análisis o intepretación de estas fuentes. En el ámbito de la creación artística, el método de análisis y de interpretación de la información se aborda de forma imbricada con la actividad creadora de los/as artistas, de acuerdo a las propias cualidades expresivas del medio artístico del que se trate (Pérez-Jofre, 2014) y, por tanto, se refiere a intervenciones artísticas. En el mapa conceptual sobre el muro de la exposición It $\mathrm{V}$, los contenidos son de tipo investigación, justificación, tipos de intervención artística y de referencias teóricas de influencia. Se cita la "INVESTIGACIÓN BASADA EN LA CREACIÓN" como metodología de investigación basada en las artes (Moreno, López-Peláez, 2016); “A partir de LO RELACIONAL y LO CONTEXTUAL", como método de investigación adoptado por la artista, de acuerdo a la teoría de Estética Relacional de Nicolas Bourriaud (Bourriaud, 2006; Prado, 2011) y al método de acción-participación (Ariño, Ferrán, 2008), respectivamente; "PROVOCACIONES ESTÉTICO ARTÍSTICAS" y “ACONTECIMIENTOS ESTÉTICO-ARTÍSTICOS" y "PROVOCACIONES ESTÉTICO ARTÍSTICAS”, como intervenciones artísticas propuestas.

El contexto teórico y artístico se refiere a una relación de conceptos, ideas, prácticas artísticas, e incluso acontecimientos históricos y personalidades que sucedieron respecto de un lugar y periodo dados, y que se consideran que son elementos característicos de este mismo. El diagrama de IC-CPPC se compone de personalidades históricas destacadas, movimientos artísticos, teorías y artistas, todos ellos interelacionados entre sí. Así, por ejemplo, se cita al filósofo Henri Focillon relacionado con la artista Lygia Clark; el artista Joaquín Torres-García aparece relacionado con el filósofo Platón, la Asociación Arte Concreto Invención y con los movimientos artísticos MADÍ y De Stijl. De modo que, en conjunto, este diagrama mural representa el contexto teórico y artístico de la muestra expuesta.

Las fuentes primarias se refieren a testimonios originales según la versión original de sus informantes. Según el soporte y el lenguaje, son de muy diverso tipo. En ItV, la artista ha elaborado los dispositivos de Mapa simbólico del miedo y Fobófono para que la población de Pereira pudiera utilizarlos para registrar en ellos sus testimonios. Así, los/as ciudadanos/as de Pereira constituyen informantes clave para la investigación artística que realiza la artista. 
Los contenidos de servicio al público se refieren a elementos informativos para dar a conocer cuáles son las posibilidades de actividades y participación para sus visitantes. En $I C-C P P C$, el dispositivo señalético del texto de presentación indicaba la procedencia de la muestra expuesta, así como incitaba al público a compartir la experiencia de la exposición en redes sociales, con citas como "EXPLORAR E INTERACTURAR" y "DESCUBRIR Y COMPARTIR", que se representaba en texto escrito junto a los iconos de las populares redes sociales de Facebook y Twitter.

Los contenidos de identificación de las obras se refieren a una información que es de tipo descriptiva, según la autoría, título, cronología, medidas y técnicas de las obras, que se encontró en los dispositivos de señalética de las cartelas y que se limitaron a contenidos de identificación de las obras.

\section{Discusión de los resultados}

La discusión de los resultados se dirige a conocer el carácter científico de las exposiciones de la muestra. El carácter científico en el área de Artes y Humanidades se refiere más bien al marco de pensamiento, sucesos y prácticas artísticas que construyen un determinado discurso de conocimiento en torno a un tema dado. Por tanto, para la discusión de los resultados se abordan estas dos preguntas: ¿se han encontrado en los resultados del análisis contenidos para un marco de pensamiento, sucesos y prácticas artísticas? En caso afirmativo, ¿cómo se ha articulado la transferencia de estos contenidos en las exposiciones de arte de la muestra?

En los resultados anteriores del análisis se han encontrado tres tipos de contenidos de los dispositivos insertos en sala que se refieren al carácter científico para el área de Artes y Humanidades, y que son: los contenidos de contexto teórico y artístico, de metodología de investigación y de fuentes primarias. En conjunto, estos tres constituyen los tres aspectos principales de la investigación en artes, de acuerdo al marco teórico, metodología y fuentes de la investigación, respectivamente. Así, desde una epistemología de la creación artística, las obras de arte constituyen los resultados de la investigación. Una exposición que integrara los contenidos de tipo crítica artística, metodología de investigación y fuentes primarias, estaría, por tanto, articulando un montaje expositivo del conjunto de la investigación artística realizada. Sin embargo, como se verá a continuación, ninguno de los dos casos de la muestra integran, a la vez, estos tres tipos de contenidos, sino que se encuentran repartidos en uno u otro caso: los contenidos de contexto teórico y artístico corresponde a la exposición IC-CPPC, mientras que los contenidos de metodología y de fuentes primarias corresponden a la exposición ItV. Por tanto, a las dos exposiciones se les reconoce cierto carácter científico en su diseño y montaje, pero no la transferencia de la investigación en las exposiciones. 


\section{Conclusiones}

En las conclusiones de este estudio se encuentra que sí es posible un carácter científico para la exposición del arte. En relación a los contenidos artísticos que se transfieren en la exposición de arte, se distinguen dos tipos diferentes de transferencia, según los siguientes:

- Una transferencia del proyecto artístico. Esta se refiere a contenidos en la exposición de arte que abordan las fases del proyecto y/o investigación artística realizada por sus autores/as. Es el caso It $V$ de la muestra, donde el dispositivo del mapa visual sintetiza la propuesta metodológica de investigación artística, y donde el Mapa simbólico del miedo y el Fobófono constituyen la recogida de datos y de evidencias de la investigación (contenidos de fuentes primarias).

- Una transferencia del contexto histórico-artístico. Esta se refiere a contenidos sobre las referencias histórico-culturales de la producción artística que se expone, según sucesos, personalidades, teorías científicas, corrientes artísticas y experiencias en la historia de vida de los/as artistas, que han sido de influencia sobre los valores de las obras. Pueden ser convergentes y/o divergentes a los discursos artísticos de las obras y de su historiografía. Este tipo de transferencia resulta apropiada para la investigación del equipo de comisariado en el proyecto de montaje de la exposición, aunque también puede abordar por parte de los/as artistas. En la muestra en este trabajo, la transferencia del contexto históricoartístico se ha articulado en el diagrama mural de $I C-C P P C$ de la muestra.

Por tanto, contextualizar las obras respecto de sus procesos de producción artística supone desplazar la exclusividad del valor estético de las obras hacia otros valores culturales. En este sentido, se destacan también otros valores de las obras, como es el valor documental, histórico e incluso social.

\section{Referencias}

Alonso, L. (2001). Museología y Museografía. 2a ed. Barcelona: Ediciones del Serbal.

Angrosino, M. (2012). Etnografia y observación participante en investigación cualitativa. Madrid: Morata.

Arañó, J. C. (2005). Estructura del conocimiento artístico. En R. Marín (ed.), Investigación en educación artística : temas, métodos y técnicas de indagación sobre el aprendizaje y la enseñanza de las artes y culturas visuales. Granada, España: Universidad de Granada, pp. 19-42. 
Ariño, M. E., Ferrán, A. (2008). Investigación-acción-participación. En C. Guinot Viciano (coord.), Métodos, técnicas y documentos utilizados en trabajo social. Navarra: Universidad de Deusto, pp. 71-78.

Ávila, A. (2003). El Arte y sus Museos. Barcelona: Editorial del Serbal.

Bourriaud, N. (2006). Estética relacional. Buenos Aires: Ed. Adriana Hidalgo.

Castro, E., Fernández, I, Pérez, M., y Criado, F. (2008). La transferencia de conocimientos desde las humanidades: posibilidades y características, ARBOR: Ciencia, Pensamiento y Cultura, 732, julio-agosto de 2008, 619-636. doi: https:// doi.org/10.3989/arbor.2008.i732.211

Conderana, J. A. (coord.). (2016). Giros epistemológicos de las artes: la creación de significado. Madrid: Ediciones Asimétricas.

Díaz, A. (2012). El taller del etnógrafo: materiales y herramientas de investigación en Etnografía. Madrid: UNED-Universidad Nacional a Distancia.

Dubé, P. (1995). Exponer para ver, exponer para conocer, Museum International, 185 (1), 4-5.

Guasch, A. M. (2016). El arte en la era de lo global: 1989-2015. Madrid: Alianza Editorial.

Hooper-Greenhill, E. (1992). Museums and the shaping of knowledge. Londres: Routledge.

Lorente, J. P. (2013). La "nueva museología" ha muerto, ¡viva la "museología crítica!”. En D. Almazán, y J. P. Lorente, Museologia, crítica y arte contemporáneo. Zaragoza, España: Universidad de Zaragoza, 13-25.

Lorente, J. P. (2008). Los museos de arte contemporáneo: Noción y desarrollo histórico. Gijón, Asturias, España: Ediciones Trea.

Moreno, M. I., López-Peláez, M. P. (coord.). (2016). Reflexiones sobre investigación artística e investigación educativa basada en las artes. Madrid: Síntesis.

Masseroni,A. (2010). Metodología ymétodos: diseños de investigación comparativa. Buenos Aires: Mnemosyne.

Medina, I., Castillo Ortiz, P. J., Álamos-Concha, P., y Rihous, B. (2017). Análisis cualitativo comparado (QCA). Madrid: Centro de Investigaciones Sociológicas.

Miles, M. B., Huberman, M. y Saldaña, J. (2014). Qualitative data analysis: a methods sourcebook. Los Angeles: SAGE. 
Pérez, P. (2007). La Insurrección Expositiva: cuando el montaje de exposiciones es creativo y divertido. Cuando la exposición se convierte en una herramienta subversiva. Gijón, Asturias, España: Ediciones Trea.

Pérez-Jofre Santesmases, I. (2014). Una reflexión sobre la creación artística como investigación. En: VV.AA., Neste universo, um rumor simultâneo [catálogo]. Vila do Conde, Portugal: Centro de Memoria de Vila do Conde, pp. 139-140.

Prado Orozco, M. (2011). Estética relacional: la experiencia estética de participación [tesis doctoral]. Departamento de Fisología, Universidad Autónoma de Barcelona, Barcelona.

Rico, J. C. (2007). Montaje de exposiciones. $3^{\text {a }}$ reimpr. Madrid: Ediciones Sílex.

Rodríguez, M. y Anglès M. (coord.). (2013) La Invención Concreta. Colección Patricia Phelps de Cisneros: reflexiones en torno a la abstracción geométrica latinoamericana y sus legados. Madrid: Departamento de Actividades Editoriales del MNCARS y TURNER.

Rojas, C. (2007). De la idea al guión: la curaduría de exposiciones en los museos de ciencia. En L. F. Rico Mansard, M. C. Sánchez, J. Tagüeña, y J. Tonda (coords.), Museología de la Ciencia: 15 años de experiencia. México D.F.: Dirección General de Divulgación de la Ciencia de la Universidad Nacional Autónoma de México, pp. 129-159.

Universidad Autónoma de Barcelona. [2018]. Transferencia del Conocimiento [en línea]: Innovación, Transferencia y Empresa. Barcelona. Disponible en: http:// www.uab.cat/web/investigar/itinerarios/innovacion-transferencia-y-empresa/ transferencia-del-conocimiento-1345667266489.html

Winckelmann, J. J. (1763, 1955). Geschichte der Kunst des Altertums. Dresde (Alemania), 1763. (Edición en español: Historia del arte en la Antiguëdad: seguida de las Observaciones sobre la arquitectura de los antiguos, Madrid: Aguilar, 1955). 